\title{
Weak Approximation over Function Fields of Curves over Large or Finite Fields
}

\author{
Yong $\mathrm{HU}^{*}$
}

\begin{abstract}
Let $K=k(C)$ be the function field of a curve over a field $k$ and let $X$ be a smooth, projective, separably rationally connected $K$-variety with $X(K) \neq \emptyset$. Under the assumption that $X$ admits a smooth projective model $\pi: \mathcal{X} \rightarrow C$, we prove the following weak approximation results: (1) if $k$ is a large field, then $X(K)$ is Zariski dense; (2) if $k$ is an infinite algebraic extension of a finite field, then $X$ satisfies weak approximation at places of good reduction; (3) if $k$ is a nonarchimedean local field and $R$ equivalence is trivial on one of the fibers $\mathcal{X}_{p}$ over points of good reduction, then there is a Zariski dense subset $W \subseteq C(k)$ such that $X$ satisfies weak approximation at places in $W$. As applications of the methods, we also obtain the following results over a finite field $k$ : (4) if $|k|>10$, then for a smooth cubic hypersurface $X / K$, the specialization map $X(K) \longrightarrow \prod_{p \in P} \mathcal{X}_{p}(\kappa(p))$ at finitely many points of good reduction is surjective; (5) if char $k \neq 2,3$ and $|k|>47$, then a smooth cubic surface $X$ over $K$ satisfies weak approximation at any given place of good reduction.
\end{abstract}

\section{Contents}

1 Introduction

2 Review of Basic Notions 5

2.1 Separably rational connectedness . . . . . . . . 5

2.2 Large fields and $R$-equivalence . . . . . . . . . . 6

3 Weak approximation over function fields 7

3.1 Geometric interpretation . . . . . . . . . . . . 7

3.2 Iterated blowup and weak approximation . . . . . . . . 8

3.3 Deformations of combs . . . . . . . . . . . . . 9

*Mathématiques, Bâtiment 425, Université Paris-Sud, 91405, Orsay Cedex, France, e-mail: yong.hu2@math.u-psud.fr 
5 Proofs of Results over Finite Fields 14

5.1 Surjectivity of specialization . . . . . . . . . . 14

5.2 Weak approximation at one place . . . . . . . 18

References

Convention. In this paper, a variety means a separated geometrically integral scheme of finite type over a field. Somewhat inconsistently, a curve means a 1-dimensional separated geometrically connected and geometrically reduced scheme of finite type over a field. The words "vector bundle" will be used interchangeably as "locally free sheaf of finite constant rank".

\section{Introduction}

Given a variety over a topological field $F$, the topology of $F$ induces a natural topology on the set of rational points. We call this topology the $F$-topology. If $K \subseteq F$ is a subfield and $X$ is a $K$-variety, the set $X(F)$ of $F$-points carries an $F$-topology via the natural identification $X(F)=X_{F}(F)$, where $X_{F}=X \times_{K} F$ is the $F$-variety obtained by base extension of the $K$-variety $X$. Let $K$ be a number field or a function field of a curve. The completion $K_{v}$ at each place $v$ of $K$ is a topological field containing $K$. The $K_{v}$-topologies will be also called $v$-adic topologies. Given a set $\Omega$ of places of $K$, we say $X$ satisfies weak approximation at places in $\Omega$ if the image of the diagonal map $X(K) \rightarrow \prod_{v \in \Omega} X\left(K_{v}\right)$ is dense, where $\prod_{v \in \Omega} X\left(K_{v}\right)$ is given the product topology of the $v$-adic topologies. The classical approximation theorem claims that the projective line $\mathbb{P}^{1}$ satisfies weak approximation at all places. In higher dimension and over the function field of a curve defined over an algebraically closed field, it is expected that weak approximation holds for separably rationally connected varieties. Roughly speaking, these are varieties containing plenty of geometric rational curves that may be deformed "very freely". (See Definition 2 for the precise definition.)

In this paper, we are interested in the following problem.

Problem 1. Let $k$ be a field, $C$ a smooth projective (geometrically connected) curve over $k, K=k(C)$ the function field of $C$, and $X$ a smooth, projective, separably rationally connected $K$-variety. Assume that $X$ has a smooth projective model, i.e., there is a surjective morphism $\pi: \mathcal{X} \rightarrow C$ from a smooth projective $k$-variety $\mathcal{X}$ to $C$ with generic fiber $X / K$. In this context, rational points of $X$ correspond to 
sections of $\pi: \mathcal{X} \rightarrow C$ and weak approximation amounts to the existence of sections passing through prescribed points in the fibers with prescribed jets.

Consider the following questions:

(EX) (Existence of sections) Is $X(K)$ nonempty? Or equivalently, does $\pi$ admit a section $\sigma: C \rightarrow \mathcal{X}$ ?

(SP) (Surjectivity of specialization map) Suppose that $\pi$ has a section $\sigma: C \rightarrow \mathcal{X}$. Let $P=\left\{p_{1}, \ldots, p_{n}\right\}$ be a finite set of closed points of $C$ such that each fiber $\mathcal{X}_{i}=\mathcal{X}_{p_{i}}$ is smooth and separably rationally connected (a point or a place with this property will be called $\boldsymbol{o f}$ good reduction). Given rational points $r_{i} \in \mathcal{X}_{i}\left(\kappa\left(p_{i}\right)\right)$ in the fibers, is there a section $s: C \rightarrow \mathcal{X}$ of $\pi$ such that $s\left(p_{i}\right)=r_{i}$ for $i=1, \ldots, n$ ?

(WA) (Weak approximation) Suppose that $X(K) \neq \emptyset$. Does the generic fiber $X$ satisfy weak approximation at a certain collection of places?

When $k$ is algebraically closed, a series of results have been established. Firstly, Kollár, Miyaoka and Mori 22] proved that question (SP) has a positive answer in this case. Later, question (EX) was solved by Graber, Harris and Starr [12] in characteristic 0 and soon generalized to positive characteristic by de Jong and Starr [6]. As for question (WA), Colliot-Thélène and Gille [3] proved that if $X$ belongs to one of some special classes of varieties, including conic bundles and del Pezzo surfaces of degree $\geq 4$, then $X$ satisfies weak approximation at all places. For arbitrary separably rationally connected varieties, Hassett and Tschinkel [15] proved weak approximation at places of good reduction, generalizing the case of cubic surfaces proved earlier by Madore [25. In the same paper, they conjectured that weak approximation at places of bad reduction also holds. This conjecture is open even for cubic surfaces. Hassett and Tschinkel [16] confirmed weak approximation for cubic surfaces with not too bad reductions. A similar result was obtained by Knecht [17] for del Pezzo surfaces of degree 2. Also inspired by the methods of Hassett and Tschinkel [16], Xu [33] proved weak approximation for del Pezzo surfaces of degree $\leq 2$ that admit a model with a certain nice property. For arbitrary del Pezzo surfaces of degree $\leq 3$ the question is still open.

As to the case where $k$ is not algebraically closed, particular results for some special fields (e.g., the reals $\mathbb{R}$ ) have been established with somewhat different methods (cf. [2], [29], [10]).

In this paper, we study the case where the ground field $k$ is a large field (cf. Definition 3) or a finite field. The approach we follow was suggested by Colliot-Thélène and Hassett in 2005. It heavily relies on the method of Hassett and Tschinkel [15] as well as on some results of Kollár and Szabó ([19], [20], [23]).

Our main results are the following. 
Theorem 1. With notation as in Problem 1, let $k$ be an infinite algebraic extension of a finite field. Assume that $X(K) \neq \emptyset$.

Then the $K$-variety $X$ satisfies weak approximation at places of good reduction.

Theorem 2. With notation as in Problem 1, let $k$ be a nonarchimedean local field. Suppose that $\pi: \mathcal{X} \rightarrow C$ has a section.

If for some point $p \in C(k), R$-equivalence ( $c f$. Definition (4) on the rational points of the fiber $\mathcal{X}_{p}$ is trivial, then there is a subset $W \subseteq C(k)$ of rational points which is Zariski dense in $C$ such that the $K$-variety $X$ satisfies weak approximation at places in $W$.

Applying the methods used in the proofs, we also obtain the following theorems.

Theorem 3. With notation as in Problem 1, let $k$ be a large field. If $X(K) \neq \emptyset$, then $X(K)$ is Zariski dense in the $K$-variety $X$.

Theorem 4. Let $k=\mathbb{F}$ be a finite field, $C$ a smooth projective (geometrically connected) curve over $k, K=k(C)$ its function field, and $\mathcal{X} \subseteq \mathbb{P}_{k}^{N} \times_{k} C$ a smooth closed subvariety such that the canonical projection $\pi: \mathcal{X} \rightarrow C$ is a surjective morphism whose generic fiber is a smooth cubic hypersurface $X \subseteq \mathbb{P}_{K}^{N}$ of dimension $\geq 2$. Suppose that $X(K) \neq \emptyset$.

Then for any finite set $P$ of closed points of good reduction on $C$ with

$$
|\kappa(p)|>10, \quad \forall p \in P,
$$

the specialization map

$$
X(K) \longrightarrow \prod_{p \in P} \mathcal{X}_{p}(\kappa(p))
$$

is surjective. In particular, if $|k|>10$, then question (SP) has a positive answer in this situation.

In proving the above theorem, we use some ideas of SwinnertonDyer, who proved an analogous result for cubic surfaces over number fields (cf. [31], Theorem 5). In particular, his method of descent from a tower of quadratic extensions of the ground field finds a significant application in our proof. Yet another important ingredient is a result of Kollár on the geometry of cubic hypersurfaces over finite fields ([21], Lemma 9.4).

As a consequence, we obtain a weak approximation theorem for cubic surfaces, which is analogous to a result of Swinnerton-Dyer ([31], Corollary to Theorem 5).

Theorem 5. Let $k=\mathbb{F}_{q}$ be a finite field of characteristic not dividing 6 and of cardinality $q>47, C$ a smooth projective (geometrically 
connected) curve over $k, K=k(C)$ its function field, and $\mathcal{X} \subseteq \mathbb{P}_{k}^{3} \times_{k} C$ a smooth closed subvariety such that the canonical projection $\pi: \mathcal{X} \rightarrow C$ is a surjective morphism whose generic fiber is a smooth cubic surface $X \subseteq \mathbb{P}_{K}^{3}$ with $X(K) \neq \emptyset$. Let $v$ be a place of good reduction corresponding to a closed point $p \in C$.

Then $X(K)$ is v-adically dense in $X\left(K_{v}\right)$. More precisely, for any $x \in \mathcal{X}_{p}(\kappa(p))$ there is an $R$-equivalence class $\mathscr{C}$ on $X(K)$ such that $\mathscr{C}$ is $v$-adically dense in the inverse image of $x$ under the specialization $\operatorname{map} X\left(K_{v}\right) \rightarrow \mathcal{X}_{p}(\kappa(p))$.

\section{Review of Basic Notions}

\subsection{Separably rational connectedness}

Definition 1 ([7], Definition 4.5). Let $X$ be a smooth variety and $r \geq 0$. An $r$-free rational curve on $X$ is a nonconstant morphism $f: \mathbb{P}^{1} \rightarrow X$ such that

$$
f^{*} T_{X} \cong \mathscr{O}_{\mathbb{P}^{1}}\left(a_{1}\right) \oplus \cdots \oplus \mathscr{O}_{\mathbb{P}^{1}}\left(a_{n}\right)
$$

with $a_{1} \geq \cdots \geq a_{n} \geq r$. (By a theorem of Grothendieck ([14], p.384, Exercise 2.6), every vector bundle over $\mathbb{P}^{1}$ is isomorphic to a direct sum $\oplus \mathscr{O}_{\mathbb{P}^{1}}\left(a_{i}\right)$ and the sequence $a_{1} \geq \cdots \geq a_{n}$ is uniquely determined by the vector bundle.) We shall say free (resp. very free) instead of 0 -free (resp. 1-free).

Definition 2 ([18], IV.3.2). Let $k$ be a field and $\bar{k}$ an algebraic closure of $k$. A smooth proper $k$-variety $X$ is called separably rationally connected if it has the following property:

(1) There is a separated integral algebraic $k$-scheme $Z$ and a morphism $g: \mathbb{P}^{1} \times_{k} Z \rightarrow X$ such that the morphism

$g^{(2)}: \mathbb{P}^{1} \times_{k} \mathbb{P}^{1} \times_{k} Z \longrightarrow X \times_{k} X ; \quad\left(t_{1}, t_{2}, z\right) \mapsto\left(g\left(t_{1}, z\right), g\left(t_{2}, z\right)\right)$

is dominant and smooth at the generic point.

When $\operatorname{dim} X>0$, this is equivalent to the following two properties:

(2) There is a very free rational curve $\mathbb{P}_{\bar{k}}^{1} \rightarrow X_{\bar{k}}$ defined over $\bar{k}$.

(3) For any finite collection of closed points $x_{1}, \ldots, x_{m} \in X_{\bar{k}}$ and any tangent directions $\xi_{i}$ at the points $x_{i}$, there is a very free curve $f: \mathbb{P}_{\bar{k}}^{1} \rightarrow X_{\bar{k}}$ defined over $\bar{k}$ such that $f$ is an unramified morphism with image containing all the $x_{i}$ as smooth points and having tangent directions $\xi_{i}$ at these points.

In property (3) of the above definition, one can take $f$ to be a closed embedding if $\operatorname{dim} X \geq 3$. For a proof of the above equivalences, see [18] IV.3.7 and 3.9, and [8], Théorème 2.2. 


\section{Example 1.}

(1) A smooth $k$-variety $X$ is separably rationally connected if it is geometrically separably unirational (i.e., over $\bar{k}$ there is a separable dominant rational map from some $\mathbb{P}_{\bar{k}}^{n}$ to $X_{\bar{k}}$ ).

(2) In characteristic 0, smooth Fano varieties are separably rationally connected (Campana, [1]). In particular, a smooth hypersurface in $\mathbb{P}^{n}$ of degree $d \leq n$ is separably rationally connected.

(3) In characteristic $p>0$, smooth cubic hypersurfaces of dimension $\geq 2$ are separably rationally connected. (Smooth cubic surfaces are geometrically rational. For the case of dimension $\geq 3$, see [5] Remarque 2.34.)

\subsection{Large fields and $R$-equivalence}

Definition 3 ([11], Chapter 10 and [27]). A field $k$ is said to be

(1) pseudo-algebraically closed (PAC), if every (geometrically integral) $k$-variety has a rational point;

(2) large, if for every (geometrically integral) $k$-variety $X$ having a smooth $k$-point, $X(k)$ is Zariski dense in $X$.

\section{Example 2.}

(1) PAC fields are large fields. Algebraic extensions of PAC (resp. large) fields are PAC (resp. large). (cf. [11], Chapter 10 and [27], Proposition 1.2.)

(2) Infinite algebraic extensions of finite fields are PAC. (cf. [11, Chapter 10.)

(3) The class of large fields contains all local fields (i.e., finite extensions of $\mathbb{R}, \mathbb{Q}_{p}$ or the field of Laurent series $\mathbb{F}_{p}((t))$ over the finite field $\mathbb{F}_{p}$ ). More generally, a field that is complete with respect to a nontrivial absolute value is large.

(4) All real closed fields and all $p$-adic closed fields (i.e., fields with absolute Galois group isomorphic to that of $\mathbb{R}$ or that of a $p$-adic field) are large (cf. [28], Theorem 7.8).

Definition 4 (Manin). Let $X$ be an algebraic scheme over a field $k$. Two rational points $x, y \in X(k)$ are said to be directly $R$-equivalent if there is a $k$-rational map $f: \mathbb{P}_{k}^{1} \rightarrow X$ such that $f(0)=x$ and $f(\infty)=y$. The $R$-equivalence relation on $X(k)$ is the equivalence relation generated by direct $R$-equivalence. We will write $X(k) / R$ for the set of $R$-equivalence classes on $X(k)$. When $X(k) / R$ has only one element, we write $X(k) / R=1$ and say that $R$-equivalence is trivial on $X$.

$R$-equivalence is closely related to separably rational connectedness. In [19], Kollár proved the finiteness of $R$-equivalence classes on separably rationally connected varieties over local fields. Later he generalized the main theorem in [19] as follows. 
Theorem 6 (Kollár, 20], Theorem 23). Let $k$ be a large field and let $X$ be a smooth, projective, separably rationally connected $k$-variety of dimension $\geq 3$. Let $n \geq 1$ and $x_{1}, \ldots, x_{n} \in X(k)$. Then the following two conditions are equivalent:

(i) There is a closed embedding $f: \mathbb{P}^{1} \rightarrow X$ that is $(n+1)$-free such that $x_{1}, \ldots, x_{n} \in f\left(\mathbb{P}^{1}(k)\right)$.

(ii) All the points $x_{i} \in X(k)$ are in the same $R$-equivalence class.

We remark that in general it may happen that there is no smooth rational curve passing through a given finite set of rational points on a variety $X$ as in the above theorem. A sufficient condition for this is that the ground field $k$ is PAC (cf. [23], Theorem 19 and [32], Corollaire 3.2). If $k=\mathbb{F}$ is a finite field, we have the following result.

Theorem 7 (Kollár and Szabó, [23], Theorems 2 and 7).

(i) There is a function $\Phi: \mathbb{N}^{3} \rightarrow \mathbb{N}$ having the following property: Let $X \subseteq \mathbb{P}^{N}$ be a smooth, projective, separably rationally connected variety over a finite field $\mathbb{F}$ of dimension $\geq 3$, and let $S \subseteq X$ be a zero-dimensional smooth subscheme. If $|\mathbb{F}|>\Phi(\operatorname{deg} X, \operatorname{dim} X, \operatorname{deg} S)$, then there is a closed embedding $f: \mathbb{P}^{1} \rightarrow X$ that is $(\operatorname{deg} S+1)$-free with image containing $S$.

(ii) There is a function $\Psi: \mathbb{N}^{2} \rightarrow \mathbb{N}$ having the following property: Let $X \subseteq \mathbb{P}^{N}$ be a smooth, projective, separably rationally connected variety over a finite field $\mathbb{F}$. If $|\mathbb{F}|>\Psi(\operatorname{deg} X, \operatorname{dim} X)$, then $X(\mathbb{F}) / R=$ 1.

In the above two theorems the hypothesis that $\operatorname{dim} X \geq 3$ is not essential. It permits us to take $f$ to be a closed embedding. If $\operatorname{dim} X=$ 2 , we can apply the theorem to $X \times_{k} \mathbb{P}^{1}$ to obtain a rational curve passing through the given points.

Remark 1. In Theorem 7 (ii), if one restricts to the case where $X \subseteq \mathbb{P}^{N}$ is a smooth cubic hypersurface of dimension $\geq 2$, then [21], Theorem 1.1 gives an even better lower bound for $|\mathbb{F}|$ : one has $X(\mathbb{F}) / R=1$ whenever $|\mathbb{F}| \geq 11$ *

\section{Weak approximation over function fields}

To establish weak approximation we use the method initiated by Hassett and Tschinkel in [15]. In this section we briefly recall their method.

\subsection{Geometric interpretation}

Let $K=k(C)$ be the function field of a smooth projective curve and let $X$ be any smooth proper variety over $K$. A result of Nagata ([26])

${ }^{*}$ The lower bound $|\mathbb{F}| \geq 8$ originally given in $[21$ should be $|\mathbb{F}| \geq 11$. See also the footnote attached to Lemma 4 . 
guarantees that $X$ always admits a proper model, i.e., an algebraic space $\pi: \mathcal{X} \rightarrow C$ that is flat and proper over $C$ with generic fiber $X$. Rational points of $X$ correspond bijectively in a natural way to sections of $\pi: \mathcal{X} \rightarrow C$.

In this paper we will assume for simplicity that $X$ is projective and admits a smooth projective model $\pi: \mathcal{X} \rightarrow C$ as in Problem 1 .

For each closed point $p \in C$, let $\mathcal{X}_{p}=\mathcal{X} \times_{C} \operatorname{Spec}(\kappa(p))$ be the fiber over $p$. Let $\widehat{\mathscr{O}}_{C, p}$ be the completed local ring at $p$, with maximal ideal $\widehat{\mathfrak{m}}_{C, p}$. If $v$ denotes the place of $K$ corresponding to $p$, then $K_{v}$ is the field of fractions of $\widehat{\mathscr{O}}_{C, p}$. Let $\widehat{C}_{p}=\operatorname{Spec}\left(\widehat{\mathscr{O}}_{C, p}\right), \mathcal{X}_{\widehat{C}_{p}}=\mathcal{X} \times{ }_{C} \widehat{C}_{p}$ and $\widehat{\pi}_{p}$ : $\mathcal{X}_{\widehat{C}_{p}} \rightarrow \widehat{C}_{p}$ the natural projection. Sections of $\widehat{\pi}_{p}$ correspond bijectively to $K_{v}$-valued points of $X$. Suppose a point $M_{v} \in X\left(K_{v}\right)$ corresponds to a section $\widehat{s}_{p}: \widehat{C}_{p} \rightarrow \mathcal{X}_{\widehat{C}_{p}}$. Then basic $v$-adic open neighborhoods of $M_{v}$ consist of those sections of $\widehat{\pi}_{p}$ which agree with

$$
\widehat{C}_{p, N}:=\operatorname{Spec}\left(\widehat{\mathscr{O}}_{C, p} / \widehat{\mathfrak{m}}_{C, p}^{N+1}\right) \hookrightarrow \widehat{C}_{p} \stackrel{\widehat{s}_{p}}{\longrightarrow} \mathcal{X}_{\widehat{C}_{p}}
$$

when restricted to $\widehat{C}_{p, N} \subseteq \widehat{C}_{p}$, for some $N \in \mathbb{N}$.

For $N \in \mathbb{N}$, we say a morphism

$$
j: \widehat{C}_{p, N}=\operatorname{Spec}\left(\widehat{\mathscr{O}}_{C, p} / \widehat{\mathfrak{m}}_{C, p}^{N+1}\right) \longrightarrow \mathcal{X}_{\widehat{C}_{p}}=\mathcal{X} \times_{C} \operatorname{Spec}\left(\widehat{\mathscr{O}}_{C, p}\right)
$$

is an $N$-jet at the closed point $p \in C$ if $\widehat{\pi}_{p} \circ j$ coincides with the natural inclusion $\widehat{C}_{p, N} \hookrightarrow \widehat{C}_{p}=\operatorname{Spec}\left(\widehat{\mathscr{O}}_{C, p}\right)$. A section of $\widehat{\pi}_{p}$ determines naturally an $N$-jet.

Thus weak approximation over function fields has a geometric reformulation as follows: for any finite set of closed points $\left\{p_{i}\right\}$ in $C$, any sections $\widehat{s}_{i}$ of $\widehat{\pi}_{p_{i}}$ and any $N \in \mathbb{N}$, there exists a section $s$ of $\pi$ such that for each $i$, the $N$-jets determined by $s$ and $\widehat{s}_{i}$ coincide.

\subsection{Iterated blowup and weak approximation}

We keep notation as above. To develop the method of Hassett and Tschinkel over an arbitrary ground field $k$, we assume that places in the consideration below are given by separable points (i.e. points with residue fields separable over $k$ ).

Let $\left\{p_{i}\right\} \subseteq C$ be a finite number of separable closed points of good reduction and let $J=\left\{j_{i}\right\}$ be $N$-jets at these points given by formal sections $\widehat{s}_{i}$ of $\widehat{\pi}_{p_{i}}: \mathcal{X}_{\widehat{C}_{p_{i}}} \rightarrow \widehat{C}_{p_{i}}$. By considering the iterated blowup associated to $J$, whose construction we will recall below, Hassett and Tschinkel reduce finding a section passing through given points with prescribed jet data to simply finding a section through given points on the iterated blowup. 
Here is the construction of the iterated blowup $\beta(J): \mathcal{X}(J) \rightarrow \mathcal{X}$ associated to $J$. It is obtained by the following sequence of blowups:

$$
\mathcal{X}(J)=\mathcal{X}_{(N)} \rightarrow \mathcal{X}_{(N-1)} \rightarrow \cdots \rightarrow \mathcal{X}_{(1)} \rightarrow \mathcal{X}
$$

where $\mathcal{X}_{(1)} \rightarrow \mathcal{X}$ is the blowup of $\mathcal{X}$ at $\left\{\widehat{s}_{i}\left(p_{i}\right)\right\}$ and for each $n=$ $1, \ldots, N-1, \mathcal{X}_{(n+1)} \rightarrow \mathcal{X}_{(n)}$ is the blowup at the points where the proper transform of $\widehat{s}_{i}$ meets the fiber over $p_{i}$.

Write $d=\operatorname{dim} X$. For each $i$ the fiber $\mathcal{X}(J)_{p_{i}}$ decomposes into irreducible components:

$$
\mathcal{X}(J)_{p_{i}}=E_{i, 0} \cup \cdots \cup E_{i, N}
$$

where

(i) $E_{i, 0}$ is the proper transform of $\mathcal{X}_{p_{i}}$, isomorphic to the blowup of $\mathcal{X}_{p_{i}}$ at the $\kappa\left(p_{i}\right)$-point $r_{i, 0}:=\widehat{s}_{i}\left(p_{i}\right)$;

(ii) $E_{i, n}$ for $n=1, \ldots, N-1$ is the blowup of $\mathbb{P}_{\kappa\left(p_{i}\right)}^{d}$ at the $\kappa\left(p_{i}\right)$ point $r_{i, n}$ where the proper transform of $\widehat{s}_{i}$ meets the fiber over $p_{i}$ of the $n$-th blowup;

(iii) $E_{i, N} \cong \mathbb{P}_{\kappa\left(p_{i}\right)}^{d}$.

Let $r_{i} \in E_{i, N} \backslash E_{i, N-1}$ denote the point where the proper transform of $\widehat{s}_{i}$ meets $E_{i, N}$. Sections $s^{\prime}$ of $\mathcal{X}(J) \rightarrow C$ with $s^{\prime}\left(p_{i}\right)=r_{i}$ yield sections of $\pi: \mathcal{X} \rightarrow C$ with $N$-jets $j_{i}$ at the points $p_{i}$. So we have the following criterion.

Proposition 1 ([15, Proposition 11). Let $X, C$ and $\pi: \mathcal{X} \rightarrow C$ be as above. Consider all data $\left(\left\{p_{i}\right\}, J=\left\{j_{i}\right\},\left\{r_{i}\right\}\right)$ consisting of a finite collection of separable closed points $\left\{p_{i}\right\} \subseteq C$ of good reduction, $N$-jets $\left\{j_{i}\right\}$ at these points and $\kappa\left(p_{i}\right)$-points $\left\{r_{i}\right\}$ on the corresponding iterated blowup $\mathcal{X}(J)$ with $r_{i} \in E_{i, N} \backslash E_{i, N-1}$.

If for every datum there exists a section $s^{\prime}$ of $\mathcal{X}(J) \rightarrow C$ with $s^{\prime}\left(p_{i}\right)=r_{i}$ for all $i$, then $X$ satisfies weak approximation at places given by separable closed points of good reduction.

\subsection{Deformations of combs}

The main steps in constructing sections through prescribed points involve techniques for smoothing combs.

Definition 5 ([15], Definition 18). Let $k$ be any field and let $\bar{k}$ be an algebraic closure of $k$. A comb with $m$ (reducible) teeth over $k$ is a projective curve $C^{*}$ over $k$ with a distinguished irreducible component $D \subseteq C^{*}$ defined over $k$ such that the following conditions hold:

(1) $D$ is a smooth (geometrically connected) curve over $k$, called the handle of the comb;

(2) $\bar{C}^{*}=C^{*} \times_{k} \bar{k}$ is the union of $\bar{D}=D \times_{k} \bar{k}$ and $m$ other curves $\bar{T}_{1}, \ldots, \bar{T}_{m}$ which are called the teeth of the comb; 
(3) each $\bar{T}_{j}$ is a chain of $\mathbb{P}^{1}$ 's;

(4) the $\bar{T}_{j}$ are disjoint with one another and each of them meets $\bar{D}$ transversally at a single point.

Let $X, C, \pi: \mathcal{X} \rightarrow C,\left\{p_{i}\right\}$ and so on be as in Proposition 1. Recall that we are assuming that the total space $\mathcal{X}$ is smooth projective and that the $p_{i}$ are separable points. Since $X$ satisfies weak approximation if and only if $X \times_{K} \mathbb{P}_{K}^{1}$ does, without loss of generality we may assume that $d=\operatorname{dim} X \geq 3$.

Suppose we are given a section $\sigma: C \rightarrow \mathcal{X}(J)$. Let $I^{\prime}$ (resp. $I^{\prime \prime}$ ) denote the set of indices with $q_{i}:=\sigma\left(p_{i}\right) \neq r_{i}\left(\right.$ resp. $\left.q_{i}=r_{i}\right)$.

Working over an algebraically closed ground field $k$, the most technical part of [15] shows that a section $C \rightarrow \mathcal{X}(J)$ passing through $r_{i}$ can be found so long as the following two tasks (A) and (B) may be accomplished:

(A). In the case $N=0$, find a comb $C^{*}$ with handle $D=\sigma(C)$ and smooth teeth $T_{1}, \ldots, T_{m}$ and a closed embedding $f: C^{*} \rightarrow \mathcal{X}$ such that the following properties hold:

(i) the teeth $T_{j}$ are very free rational curves contained in distinct general fibers of $\pi$;

(ii) for each $i \in I^{\prime}$, there is a tooth $T_{i}$ with image containing $r_{i}$ as a smooth point;

(iii) if $\mathfrak{r}$ denotes the set of points on $C^{*}$ mapping to the $r_{i}, i \in I=$ $I^{\prime} \cup I^{\prime \prime}$ and $N_{f}=N_{C^{*} / \mathcal{X}}$ denotes the normal bundle of $C^{*}$ in $\mathcal{X}$, then the restriction of $N_{f} \otimes \mathscr{O}_{C^{*}}(-\mathfrak{r})$ to every irreducible component of $C^{*}$ is generated by global sections and has no higher cohomology.

(B). In the case $N \geq 1$, assume $q_{i} \in E_{i, N} \backslash E_{i, N-1}$. Find a comb $C^{*}$ with handle $D=\sigma(C)$ and reducible teeth $T_{1}, \ldots, T_{m}$ and a closed embedding $f: C^{*} \rightarrow \mathcal{X}(J)$ having the following properties:

(i) for each $i \in I^{\prime}$, there is a tooth $T_{i}$ mapped into $\mathcal{X}(J)_{p_{i}}$ and containing $r_{i}$;

(ii) for each $i \in I^{\prime}, f\left(C^{*}\right)$ contains $r_{i}$ as a smooth point, so there is a unique irreducible component $T_{i, N} \subseteq C^{*}$ with image containing $r_{i}$;

(iii) the remaining teeth $T_{j}, j=\left|I^{\prime}\right|+1, \ldots, m$ are very free rational curves contained in distinct general fibers of $\mathcal{X}(J) \rightarrow C$;

(iv) if $\mathfrak{r}$ denotes the set of points on $C^{*}$ mapping to the $r_{i}, i \in I=$ $I^{\prime} \cup I^{\prime \prime}$ and $N_{f}=N_{C^{*} / \mathcal{X}(J)}$ denotes the normal bundle of $C^{*}$ in $\mathcal{X}(J)$, then the restriction of $N_{f} \otimes \mathscr{O}_{C^{*}}(-\mathfrak{r})$ to every irreducible component of $C^{*}$ is generated by global sections and has no higher cohomology.

In [15], (A) and (B) are done in Lemmas 25 and 26, assuming of course the generic fiber $X$ is separably rationally connected. The desired section is a smooth deformed curve of the comb $C^{*}$, which is obtained by applying [15], Proposition 24. 
The key condition here is the vanishing of the higher cohomology of the twisted normal sheaf $N_{f} \otimes \mathscr{O}_{C^{*}}(-\mathfrak{r})$. We consider this condition on each irreducible component of $C^{*}$. For a component in a tooth $T_{j}$, the condition is easier to verify since such a curve is usually a very free curve. The difficult part is the restriction to the handle $D=\sigma(C)$. Fortunately, the sheaf $N_{f} \otimes \mathscr{O}_{D}$ has a nice interpretation as a kind of elementary transform of $N_{\sigma}$, where $N_{\sigma}$ denotes the normal sheaf of $D$ in $\mathcal{X}(J)$. Let $D^{c}$ be the closure of $C^{*} \backslash D$ in $C^{*}$ and $S=D \cap D^{c}$ the locus where the handle $D$ meets the teeth. For each $q_{i} \in S$, let $\xi_{i} \subseteq N_{\sigma} \otimes \kappa\left(q_{i}\right)$ denote the subspace determined by the tangent direction $T_{D^{c}, q_{i}}$. It turns out that $N_{f} \otimes \mathscr{O}_{D}$ is the so-called sheaf of rational sections of $N_{\sigma}$ having at most a simple pole at each $q_{i}$ in the direction $\xi_{i}$ and regular elsewhere (cf. [12], Lemma 2.6).

A key lemma needed is the following.

Lemma 1 ([12], Lemma 2.5). Let $C$ be a smooth curve over an algebraically closed field, $\mathscr{V}$ a vector bundle on $C$ and let $l \geq 1$ be a positive integer. Then for sufficiently large $m$ there exist general points $q_{1}, \ldots, q_{m} \in C$ and one-dimensional subspaces $\xi_{i} \subseteq \mathscr{V} \otimes \kappa\left(q_{i}\right)$ such that for any $l$ points $w_{1}, \ldots, w_{l} \in C$ the sheaf $\mathscr{V}^{\prime}\left(-w_{1}-\cdots-w_{l}\right)$ is generated by global sections and

$$
H^{1}\left(C, \mathscr{V}^{\prime}\left(-w_{1}-\cdots-w_{l}\right)\right)=0,
$$

where $\mathscr{V}^{\prime}$ denotes the sheaf of rational sections of $\mathscr{V}$ that have at most a simple pole at each $q_{i}$ in the direction $\xi_{i}$ and regular elsewhere.

Note that the smallest value for $m$ depends on the number $l$ and the vector bundle $\mathscr{V}$.

Lemma 1 applied to the normal bundle $\mathscr{V}=N_{\sigma}$ on $D$ shows that the vanishing of higher cohomology required in the tasks (A) and (B) may be guaranteed by adding sufficiently many teeth to the comb $C^{*}$.

Now we want to deal with the case where $k$ is not necessarily algebraically closed and we want to produce a deformed curve defined over $k$. We face two problems.

(D.1) We should be able to construct the comb $C^{*}$ over $k$.

First consider task (A). Suppose that we want to add teeth in the fibers over separable closed points $p_{i} \in C$. If we are able to find in each fiber a very free curve $T_{i}$ defined over $\kappa\left(p_{i}\right)$, then the union $\cup_{i} T_{i} \cup \sigma(C)$ considered as a $k$-scheme gives us a comb defined over $k$. Unlike the case of algebraically closed ground field, the existence of a very free curve defined over the ground field in a separably rationally connected fiber is not a priori evident, and if we moreover require the very free curve to pass through two prescribed points, this may be even impossible. In the next section we shall use the results of Kollár and Szabó on the triviality of $R$-equivalence to solve this problem. 
The situation for (B) is essentially the same. The difference is that the fiber $\mathcal{X}(J)_{p_{i}}$ becomes a bit more complicated. However, by a careful inspection on the construction of reducible teeth in [15], we see that for each reducible tooth $T_{i}$ all but one of its components $T_{i, 0}, \ldots, T_{i, N}$ are just lines connecting two $\kappa\left(p_{i}\right)$-points and the last one $T_{i, 0}$ is a very free curve passing through one $\kappa\left(p_{i}\right)$-point in the piece $E_{i, 0}$, which is the blowup of $\mathcal{X}_{p_{i}}$ at a $\kappa\left(p_{i}\right)$-point. Separably rational connectedness and the set of $R$-equivalence classes are both birationally invariant for smooth projective varieties (cf. [18, IV.3.3 and [4], p.195, Proposition 10). So if a property determined by separably rational connectedness or $R$-equivalence holds for $\mathcal{X}_{p_{i}}$, then the same should hold for $E_{i, 0}$. In particular, if the same thing can be done in $\mathcal{X}_{p_{i}}$, then the curve $T_{i, 0}$ in $E_{i, 0}$ may be constructed over $\kappa\left(p_{i}\right)$. So it makes no essential difference in fulfilling tasks $(\mathrm{A})$ and $(\mathrm{B})$.

(D.2) We should be able to find deformations of $C^{*}$ that are defined over $k$.

Having constructed a comb $C^{*}$ defined over $k$ with good properties as stated in (A) or (B), by applying the same argument as in [15] we see that the parameter space $M$ of curves in $\mathcal{X}(J)$ passing through the separable points $\left\{r_{i}\right\}$ is smooth at the $k$-point $\left[C^{*}\right]$ and there are nearby deformed curves defined over $\bar{k}$ that are smooth. The final step is to show the existence of a $k$-rational point in the parameter space. When the field $k$ is large or finite, we have some tools to do this as we shall now see.

\section{Proofs of Results over Large Fields}

Proposition 2. With notation as in Problem 1, let $k$ be a large field. Consider question (SP). Suppose that the closed points $p_{1}, \ldots, p_{n}$ are separable and that the point $r_{i} \in \mathcal{X}_{i}\left(\kappa\left(p_{i}\right)\right)$ lies in the same $R$-equivalence class as $q_{i}=\sigma\left(p_{i}\right) \in \mathcal{X}_{i}\left(\kappa\left(p_{i}\right)\right)$ for every $i=1, \ldots, n$.

Then there exists a section $s: C \rightarrow \mathcal{X}$ of $\pi$ such that $s\left(p_{i}\right)=r_{i}$ for $i=1, \ldots, n$.

Proof. In each fiber $\mathcal{X}_{i}=\mathcal{X}_{p_{i}}$, the two rational points $r_{i}$ and $q_{i}=\sigma\left(p_{i}\right)$ are $R$-equivalent by hypothesis. Thus Kollár's theorem (Theorem 6) implies that there is a very free curve $f_{i}: \mathbb{P}^{1} \rightarrow \mathcal{X}_{i}$ defined over $\kappa\left(p_{i}\right)$ such that $q_{i}, r_{i} \in f_{i}\left(\mathbb{P}^{1}\left(\kappa\left(p_{i}\right)\right)\right)$. On the other hand, the smooth curve $C$ has sufficiently many separable closed points. So we can pick as many good points as we need and construct teeth in the corresponding fibers to get a comb $C^{*}$ defined over $k$ satisfying all the properties in task (A). The comb $C^{*}$ then has smooth curves as nearby deformations, and since $\left[C^{*}\right]$ is a smooth $k$-point in the parameter space, the property of large fields implies that there exists a deformed smooth curve that is 
defined over $k$. The problems (D.1) and (D.2) described in $\S 3$ may be thus solved. We then get a section $s$ passing through the points $r_{i}$.

Proof of Theorem 1. Let $U \subseteq C$ be the open subset consisting of points of good reduction. (Such an open subset exists by [18], IV.3.11.) Note that $k$ is perfect so that every point is separable. Let $p_{1}, \ldots, p_{n}$ be closed points lying in $U$.

The theorem of Kollár and Szabó (Theorem 7 (ii)) implies that $R$ equivalence on a smooth separably rationally connected variety over an infinite algebraic extension of a finite field is trivial. So we have $\mathcal{X}_{p_{i}}\left(\kappa\left(p_{i}\right)\right) / R=1$. A similar argument as in the proof of Proposition 2 shows that a comb $C^{*}$ defined over $k$ may be constructed satisfying all the required properties in task (A) or (B). Since $k$ is a large field, the problem (D.2) may also be solved. This means that the comb $C^{*}$ admits smooth deformed curves defined over $k$, which give rise to sections on the iterated blowup with the required property. Weak approximation at places in $U$ is thus proved for the generic fiber $X$.

Proof of Theorem [. By a result of Kollár ([20], Theorem 3), the function

$$
C(k) \longrightarrow \mathbb{N}, \quad c \mapsto\left|\mathcal{X}_{c}(k) / R\right|
$$

is upper semi-continuous for the $k$-topology. So there is a nonempty open subset $W$ of $C(k)$ (for the $k$-topology) such that $\mathcal{X}_{c}(k) / R=1$ for all $c \in W$. For any nonempty Zariski open subset $U$ of $C, U(k)$ is dense in $C(k)$ for the $k$-topology. Hence $W \cap U(k) \neq \emptyset$, showing that $W$ is Zariski dense in $C$. The same argument as in the proof of Theorem 1 proves weak approximation at places in $W$.

Remark 2. In Theorem 2, the hypothesis on the existence of a point $p \in C(k)$ with $\mathcal{X}_{p}(k) / R=1$ is satisfied if there is a point $p \in C(k)$ such that $\mathcal{X}_{p}$ has a smooth separably rationally connected reduction over the residue field $\mathbb{F}$ of $k$ and if the residue field $\mathbb{F}$ has sufficiently large cardinality (cf. [23], Theorem 8).

The following lemma is an easy consequence of a theorem of Kollár.

Lemma 2. Let $X$ be a smooth, projective, separably rationally connected variety over a large field $k$. Suppose that $X(k) \neq \emptyset$. Then for every $x \in X(k)$, there is a subset $W \subseteq X(k)$ that is Zariski dense in $X$ with the following property: for every $y \in W$ there is a very free curve $f_{x y}: \mathbb{P}^{1} \rightarrow X$ defined over $k$ such that $x, y \in f_{x y}\left(\mathbb{P}^{1}(k)\right)$.

Proof. In [19], Theorem 1.4, Kollár proved that there exists a very free curve $f_{x}: \mathbb{P}^{1} \rightarrow X$ defined over $k$ sending a rational point $0 \in \mathbb{P}^{1}$ to $x$. Let $\operatorname{Hom}\left(\mathbb{P}^{1}, X ; 0 \mapsto x\right)$ be the Hom-scheme parametrizing morphisms 
$\mathbb{P}^{1} \rightarrow X$ sending 0 to $x$. By [7], p.91, Proposition 4.8, the evaluation map

$$
e: \quad \mathbb{P}^{1} \times \operatorname{Hom}\left(\mathbb{P}^{1}, X ; 0 \mapsto x\right) \longrightarrow X, \quad(t, g) \mapsto g(t)
$$

is smooth at any rational point $\left(t,\left[f_{x}\right]\right)$ with $t \neq 0$. In particular, $e$ is dominant. Therefore, for every nonempty open subset $U$ of $X$, we can find a very free curve $f: \mathbb{P}^{1} \rightarrow X$ defined over $k$ sending a rational point of $\mathbb{P}^{1}$ into $U$ with $f(0)=x$.

Proof of Theorem 3. Let $\sigma: C \rightarrow \mathcal{X}$ be a section of $\pi: \mathcal{X} \rightarrow C$. Let $Z$ be a proper closed subset of $X$. Its closure in $\mathcal{X}$ is a proper closed subset $\mathcal{Z}$ of $\mathcal{X}$. There is a nonempty open subset $U$ of $C$ such that for every $c \in U$, the fiber $\mathcal{X}_{c}$ is smooth separably rationally connected and $\mathcal{X}_{c} \cap \mathcal{Z} \neq \mathcal{X}_{c}$. Choose a separable closed point $p \in U$. By Lemma 2, there is a point $r \in \mathcal{X}_{p}(\kappa(p)) \backslash \mathcal{Z}$ which is directly linked to $q=\sigma(p)$ by a very free rational curve defined over $\kappa(p)$. Proposition 2 implies that there is a section $s: C \rightarrow \mathcal{X}$ such that $s(p)=r$. This section gives a $K$-rational point of $X$ which does not lie in $Z$. Since $Z$ is arbitrary, it follows that $X(K)$ is Zariski dense in $X$.

\section{$5 \quad$ Proofs of Results over Finite Fields}

\subsection{Surjectivity of specialization}

Proposition 3. With notation as in Problem 1 , let $k=\mathbb{F}$ be a finite field. Let $S \subseteq C$ be the closed subset consisting of points of bad reduction for the model $\pi: \mathcal{X} \rightarrow C$. Assume that $\pi$ has a section $\sigma: C \rightarrow \mathcal{X}$. We fix closed embeddings

$$
C \hookrightarrow \mathbb{P}_{k}^{3}, \quad \mathcal{X} \hookrightarrow \mathbb{P}_{k}^{N} \times_{k} C \hookrightarrow \mathbb{P}_{k}^{N} \times_{k} \mathbb{P}_{k}^{3} \hookrightarrow \mathbb{P}_{k}^{M}
$$

where $\mathbb{P}_{k}^{N} \times_{k} \mathbb{P}_{k}^{3} \hookrightarrow \mathbb{P}_{k}^{M}$ is the Segre embedding. We denote by $\operatorname{deg} C$ the degree of $C$ in $\mathbb{P}_{k}^{3}$, $\operatorname{deg} \sigma(C)$ and $\operatorname{deg} \mathcal{X}$ the degrees of $\sigma(C)$ and $\mathcal{X}$ in $\mathbb{P}_{k}^{M}$, and $\operatorname{deg} X$ the degree of $X$ with respect to the embedding

$$
X=\mathcal{X} \times_{C} K \hookrightarrow \mathbb{P}_{K}^{N}=\left(\mathbb{P}_{k}^{N} \times_{k} C\right) \times_{C} K
$$

There is a lower bound

$$
B=B\left(N_{\sigma},|S|, \operatorname{deg}_{C} P, \operatorname{deg} C, \operatorname{deg} \sigma(C), \operatorname{deg} X, \operatorname{dim} X\right)
$$

for the cardinality $|\mathbb{F}|$, depending on the normal bundle $N_{\sigma}$ of the section $\sigma$, the cardinality $|S|$, the number $\operatorname{deg}_{C} P=\sum_{p \in P}[\kappa(p): k]$ and the geometric invariants $\operatorname{deg} C, \operatorname{deg} \sigma(C), \operatorname{deg} X$ and $\operatorname{dim} X$, such that the answer to question (SP) is yes whenever $|\mathbb{F}|>B$. 
Proof. According to the method we described in $\S 3$, we want to first construct a comb $C^{*}$ such that the conditions in task (A) hold. When $|\mathbb{F}|$ is greater than a lower bound in terms of $\operatorname{deg} X$ and $\operatorname{dim} X$, by the theorem of Kollár and Szabó (Theorem 7 (i)), in any fiber of $\mathcal{X} \rightarrow C$ over a point $p \in C$ of good reduction there always exists a very free curve defined over $\kappa(p)$ and passing through two given $\kappa(p)$-points. This implies that the comb $C^{*}$ may be constructed over $\mathbb{F}$ when $|\mathbb{F}|$ is large enough.

In addition to the teeth given by very free curves in fibers over some points of $P$, many other teeth have to be added to the comb $C^{*}$ such that the condition on the vanishing of higher cohomology in task (A) holds. Let $m$ be the number of these teeth. The smallest value for $m$ depends on the normal bundle $N_{\sigma}$ and the number $\operatorname{deg}_{C} P$, as we remarked after the statement of Lemma 1. By the Lang-Weil estimate ([24], Theorem 1), we may assume that $|\mathbb{F}|$ is greater than a lower bound depending on $\operatorname{deg} C, \operatorname{deg}_{C} P$ and $|S|$ so that we can find $m \mathbb{F}$-rational points outside $P \cup S$. We then construct the $m$ teeth in fibers over these points. The total number of teeth of the comb $C^{*}$ is at $\operatorname{most}_{\operatorname{deg}} P+m$. By [23], Theorem 16 and the Lang-Weil estimate (cf. [23], p.258, Proof of Theorem 16 implies Theorem 2), we may choose each tooth with degree bounded from above in terms of $\operatorname{dim} X, \operatorname{deg} X$. Thus the degree $d$ of $C^{*}$ is bounded from above in terms of $\operatorname{deg}_{C} P, m, \operatorname{dim} X, \operatorname{deg} X$ and $\operatorname{deg} \sigma(C)$.

In the parameter space $M_{d}$ of degree $d$ curves in $\mathcal{X}$ containing $\left\{r_{i}\right\}$, the comb $C^{*}$ corresponds to a smooth $\mathbb{F}$-rational point $\left[C^{*}\right]$. Choose a smooth geometrically irreducible curve $T$ in $M_{d}$ passing through $\left[C^{*}\right]$ such that deformations given by points in $W=T \backslash\left\{\left[C^{*}\right]\right\}$ are all smooth curves. Then $W$ is contained in a unique geometrically irreducible component $W^{\prime}$ of the subspace $H_{d}$ parametrizing smooth degree $d$ curves in $\mathcal{X}$ containing $\left\{r_{i}\right\}$. By [23], Proposition 20, the basic projective invariants of $W^{\prime}$ are bounded in terms of $\operatorname{dim} \mathcal{X}, \operatorname{deg} \mathcal{X}, d$ and $\operatorname{deg}_{C} P$. In our situation, $\operatorname{dim} \mathcal{X}$ and $\operatorname{deg} \mathcal{X}$ can be expressed in terms of $\operatorname{dim} X, \operatorname{deg} C$ and $\operatorname{deg} X$. Taking into account all these things, we finally get, using the Lang-Weil theorem, a lower bound

$$
B=B\left(N_{\sigma},|S|, \operatorname{deg}_{C} P, \operatorname{deg} C, \operatorname{deg} \sigma(C), \operatorname{deg} X, \operatorname{dim} X\right)
$$

having the property that $W^{\prime}(\mathbb{F}) \neq \emptyset$ whenever $|\mathbb{F}|>B$. Let $\mathcal{C}_{w}$ be a deformed curve corresponding to a rational point of $W^{\prime}$. Deformed curves given by points of $W$ have the same numerical properties as $C^{*}$, so does the curve $\mathcal{C}_{w}$. In particular, the intersection number of $\mathcal{C}_{w}$ with a general fiber of $\mathcal{X} \rightarrow C$ is one. Therefore, the curve $\mathcal{C}_{w}$ gives a section $s: C \rightarrow \mathcal{X}$ as we want.

A drawback of the result in Proposition 3 is that the lower bound $B$ is ineffective and depends on many non-intrinsic objects. However, 
from the above proof we see that the dependence of $B$ on the normal bundle $N_{\sigma}$ relies only on its cohomological property, so the value of $B$ will not change when we go over to an extension of the ground field $\mathbb{F}$.

Let us now start the proof of Theorem 4. Basically, it amounts to showing that it is really possible to come down to the ground field $\mathbb{F}$ from a big enough extension $F_{n}$ over which the result may be guaranteed by Proposition 3 . Using geometry of cubic hypersurfaces we will do this with $F_{n} / \mathbb{F}$ a tower of quadratic extensions. The basic ideas go back to Swinnerton-Dyer, who obtained similar results for cubic surfaces over number fields (cf. [31], Theorem 5 and its corollary).

Let $V \subseteq \mathbb{P}_{k}^{N}$ be a cubic hypersurface over a field $k$ and let $k_{1} / k$ be a quadratic extension. Denote by $\sigma$ the nontrivial element in the Galois $\operatorname{group} \operatorname{Gal}\left(k_{1} / k\right)$. We define a "dashed arrow"

$$
V\left(k_{1}\right) \rightarrow V(k)
$$

as follows: For a point $v_{1} \in V\left(k_{1}\right)$, the line $\ell\left(v_{1},{ }^{\sigma} v_{1}\right)$ joining $v_{1}$ and its conjugate point ${ }^{\sigma} v_{1}$ will generally intersect $V$ at only one more point $v_{0}$, which is $k$-rational. Whenever this is well-defined, we associate to $v_{1} \in V\left(k_{1}\right)$ the third intersection point $v_{0} \in V(k)$ of the line $\ell\left(v_{1},{ }^{\sigma} v_{1}\right)$ and $V$. In what follows, we will always mean the dashed arrow is defined at $v_{1}$ and sends it to $v_{0}$ when we write " $v_{1}-\rightarrow v_{0}$ ".

Lemma 3. Let $A$ be a discrete valuation ring with field of fractions $K$ and residue field $F$. Let $X_{K} \subseteq \mathbb{P}_{K}^{N}$ be a smooth cubic hypersurface and $\mathcal{X}_{A} \subseteq \mathbb{P}_{A}^{N}$ its scheme-theoretic closure in $\mathbb{P}_{A}^{N}$. Suppose that the special fiber $X_{F} \subseteq \mathbb{P}_{F}^{N}$ is a smooth cubic hypersurface. Let $F_{1} / F$ be a quadratic extension and $K_{1} / K$ an unramified quadratic extension having residue field $F_{1} / F$. Then the diagram

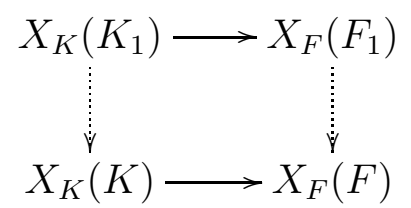

is commutative in the following sense:

If $x_{1} \in X_{K}\left(K_{1}\right)$ specializes to $\widetilde{x}_{1} \in X_{F}\left(F_{1}\right)$ and $\widetilde{x}_{1} \rightarrow \widetilde{x}_{0} \in X_{F}(F)$, then the dashed arrow $X_{K}\left(K_{1}\right) \rightarrow X_{K}(K)$ is defined at $x_{1}$ and the image $x_{0} \in X_{K}(K)$ of $x_{1}$ specializes to $\widetilde{x}_{0} \in X_{F}(F)$.

Proof. Let $\sigma$ be the nontrivial element of the Galois group $\operatorname{Gal}\left(K_{1} / K\right)=$ $\operatorname{Gal}\left(F_{1} / F\right)$. The hypothesis $\widetilde{x}_{1} \rightarrow \widetilde{x}_{0}$ means that the $F$-line $L_{F}=$ $\ell\left(\widetilde{x}_{1},{ }^{\sigma} \widetilde{x}_{1}\right)$ joining $\widetilde{x}_{1}$ and ${ }^{\sigma} \widetilde{x}_{1}$ is not contained in $X_{F}$ and the intersection $\ell\left(\widetilde{x}_{1},{ }^{\sigma} \widetilde{x}_{1}\right) \cap X_{F}$ has exactly 3 points, the third being $\widetilde{x}_{0} \in X_{F}(F)$. Since $K_{1} / K$ is unramified, it is easily verified that ${ }^{\sigma} x_{1}$ specializes to ${ }^{\sigma} \widetilde{x}_{1}$. Let $L_{K}=\ell\left(x_{1},{ }^{\sigma} x_{1}\right)$ be the $K$-line joining $x_{1}$ and ${ }^{\sigma} x_{1}$. Let $\mathcal{L}_{A} \subseteq \mathbb{P}_{A}^{N}$ be its scheme-theoretic closure in $\mathbb{P}_{A}^{N}$. 
Claim. $\mathcal{L}_{A}$ is an $A$-line in $\mathbb{P}_{A}^{N}$.

We leave aside the proof of the claim for a moment and continue the proof of the lemma.

The special fiber $\mathcal{L}_{A} \times{ }_{A} F$ is thus a line containing $\widetilde{x}_{1}$ and ${ }^{\sigma} \widetilde{x}_{1}$, whence $\mathcal{L}_{A} \times_{A} F=L_{F}=\ell\left(\widetilde{x}_{1},{ }^{\sigma} \widetilde{x}_{1}\right)$. We have $L_{K} \nsubseteq X_{K}$ since $L_{F} \nsubseteq$ $X_{F}$. Hence $L_{K} \cap X_{K}=\left\{x_{1},{ }^{\sigma} x_{1}, x_{0}\right\}$ with $x_{0} \in X_{K}(K)$, showing that $x_{1} \rightarrow x_{0}$. To prove $x_{0}$ specializes to $\widetilde{x}_{0}$, it suffices to show that the scheme-theoretic closure of $Z_{K}:=L_{K} \cap X_{K} \subseteq \mathbb{P}_{K}^{N}$ in $\mathbb{P}_{A}^{N}$ is equal to $\mathcal{Z}_{A}=\mathcal{L}_{A} \cap \mathcal{X}_{A} \subseteq \mathbb{P}_{A}^{N}$. By [13], (IV.2.8.5), we need only show that $\mathcal{Z}_{A}$ is $A$-flat. Note that $\mathcal{Z}_{A} \rightarrow \operatorname{Spec} A$ is proper and both the generic fiber $Z_{K} / K$ and the special fiber $Z_{F}=L_{F} \cap X_{F} / F$ are finite. By Chevalley's theorem, $\mathcal{Z}_{A}$ is finite over $A$. Since the generic fiber $Z_{K} / K$ and the special fiber $Z_{F} / F$ have the same length, $\mathcal{Z}_{A}$ is flat over $A$ by [14], p.174, Lemma 8.9.

We finish by giving the proof of our claim. This is essentially an easy consequence of [13], (IV.2.8.1.1).

The embedding $L_{K} \subseteq \mathbb{P}_{K}^{N}$ corresponds to a surjective $K$-homomorphism $\varphi: K^{N+1} \rightarrow K^{2}$. Let $M$ be the image of the composite map $A^{N+1} \hookrightarrow$ $K^{N+1} \stackrel{\varphi}{\longrightarrow} K^{2}$ and let $\varphi_{A}: A^{N+1} \rightarrow M$ be the induced map. Then the surjection $\varphi_{A}: A^{N+1} \rightarrow M$ corresponds to the embedding $\mathcal{L}_{A} \subseteq \mathbb{P}_{A}^{N}$. We need prove that $M$ is free of rank 2 over $A$. In fact, there is an induced commutative diagram with exact rows

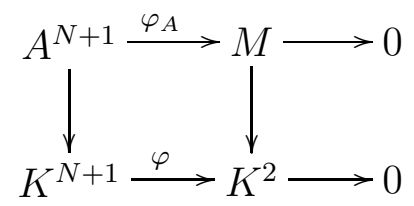

As an $A$-submodule of $K^{2}, M$ is torsion-free and hence free over the discrete valuation ring $A$. The natural homomorphism $M \otimes_{A} K \rightarrow K^{2}$ is bijective, whence $\operatorname{rank}_{A} M=2$. This completes the proof.

We shall now make use of the following lemma due to Kollár.

Lemma 4 ([21], Lemma 9.4). Let $\mathbb{F}_{q}$ be a finite field of cardinality $q \geq 11$ Then for any smooth cubic hypersurface $X \subseteq \mathbb{P}^{N}$ defined over $\mathbb{F}_{q}$ with $N \geq 2$, the dashed arrow

$$
X\left(\mathbb{F}_{q^{2}}\right) \rightarrow X\left(\mathbb{F}_{q}\right)
$$

is surjective, i.e., for every $p \in X\left(\mathbb{F}_{q}\right)$, there is a point $x \in X\left(\mathbb{F}_{q^{2}}\right) \backslash$ $X\left(\mathbb{F}_{q}\right)$ such that the line $\ell\left(x,{ }^{\sigma} x\right)$ joining $x$ and its conjugate point ${ }^{\sigma} x$ satisfies

$$
\ell\left(x,{ }^{\sigma} x\right) \cap X=\left\{p, x,{ }^{\sigma} x\right\} .
$$

\footnotetext{
${ }^{\dagger}$ In [21, the proof of Lemma 9.4 holds only for $q \geq 11$.
} 
Proof of Theorem 4. We consider the case where $P=\{p\}$ consists of a single point. The general case may be treated in the same way without essential difference.

Let $\kappa=\kappa(p)$ be the residue field of the point. We fix an algebraic closure $\overline{\mathbb{F}}$ of $\mathbb{F}$ and let $F_{n}, \kappa_{n}, n \geq 0$ be the subfields of $\overline{\mathbb{F}}$ determined by the following conditions:

$$
F_{0}=\mathbb{F}, \kappa_{0}=\kappa, F_{n} \subseteq \kappa_{n}, \text { and }\left[F_{n+1}: F_{n}\right]=\left[\kappa_{n+1}: \kappa_{n}\right]=2,
$$

for all $n \geq 0$. Let $K_{n}=F_{n}\left(C_{F_{n}}\right)$ be the function field of the curve $C_{F_{n}}=C \times_{\mathbb{F}} F_{n}$ defined over $F_{n}$. Then for each $n$ the following diagram is commutative in the sense described as in Lemma 3:

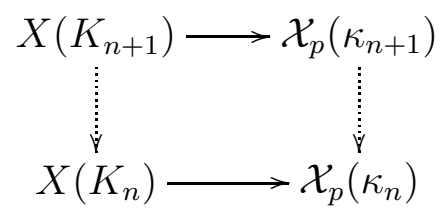

Suppose that $|\kappa(p)|>\phi(N)$. Then, by Lemma 4, starting from any point $r_{0} \in \mathcal{X}_{p}\left(\kappa_{0}\right)$ we can find successively a sequence of points $r_{i} \in$ $\mathcal{X}_{p}\left(\kappa_{i}\right)$ such that for each $i, r_{i+1} \rightarrow r_{i}$ via the dashed arrow $\mathcal{X}_{p}\left(\kappa_{i+1}\right) \rightarrow$ $\mathcal{X}_{p}\left(\kappa_{i}\right)$. Due to Proposition 3, we may choose $n$ big enough so that the specialization map $X\left(K_{n+1}\right) \rightarrow \mathcal{X}_{p}\left(\kappa_{n+1}\right)$ is surjective. Pick a point $s_{n+1} \in X\left(K_{n+1}\right)$ that specializes to $r_{n+1} \in \mathcal{X}_{p}\left(\kappa_{n+1}\right)$. By Lemma 3, we obtain points $s_{i} \in X\left(K_{i}\right)$ for $i=n+1, n \ldots, 0$ such that

$$
s_{n+1}-\rightarrow s_{n}-\rightarrow \cdots \cdots-\rightarrow s_{1}-\rightarrow s_{0}
$$

and each $s_{i}$ specializes to $r_{i}$. In particular, there exists a point $s_{0} \in$ $X(K)$ which specializes to $r_{0} \in \mathcal{X}_{p}(\kappa(p))$.

\subsection{Weak approximation at one place}

Our proof of Theorem 5 follows the method of Swinnerton-Dyer. In particular, the following result due to him will be needed.

Theorem 8 ([31], Theorem 4). Let $K$ be a global field, $K_{v}$ the completion of $K$ at a nonarchimedean place $v$ and $k$ the residue field of $v$. Let $V \subseteq \mathbb{P}_{K}^{3}$ be a smooth cubic surface whose reduction $\widetilde{V} \subseteq \mathbb{P}_{k}^{3}$ at $v$ is also smooth. Let $\widetilde{P} \in \widetilde{V}(k)$ be the reduction of a point $P \in V(K)$.

Suppose that there is a point $R \in V(K)$ whose reduction $\widetilde{R} \in \widetilde{V}(k)$ has the following properties:

(1) the line $\widetilde{P} \widetilde{R}$ intersects $\widetilde{V}$ at exactly three distinct points;

(2) no geometric line on $\widetilde{V}$ passes through $\widetilde{R}$;

(3) letting $\gamma=T_{\widetilde{R}} \widetilde{V} \cap \widetilde{V}$, there exist two distinct points $t_{1}, t_{2} \in \gamma(k)$ such that for each $i=1,2, t_{i} \neq \widetilde{R}$ and the line $\widetilde{P} t_{i}$ intersects $\widetilde{V}$ at three distinct points and

$$
T_{t_{1}} \gamma \cap T_{t_{2}} \gamma \cap T_{\widetilde{P}} \widetilde{V}=\emptyset
$$


Then there is an $R$-equivalence class $\mathscr{C}$ in $V(K)$ such that for every point $Q^{*} \in V\left(K_{v}\right)$ that specializes to $\widetilde{P} \in \widetilde{V}(k)$ and every $v$-adic open neighborhood $\mathscr{U}_{v}$ of $Q^{*}$, one has $\mathscr{C} \cap \mathscr{U}_{v} \neq \emptyset$.

Swinnerton-Dyer stated the above theorem for a number field $K$. But his proof works for function fields as well. (In his paper [31], Swinnerton-Dyer deduced this theorem from his Lemma 8 and Theorem 3. In proving these two results the key ingredients have been nonarchimedean analysis plus some geometric arguments involving tangent spaces and intersection theory, which do not actually depend on special properties of number fields as one may verify with more or less patience.)

In the proof of the next result, some geometric arguments have been used in [31] and [21]. The idea being very similar, here we still include a proof because of a slightly greater generality and some useful explicit estimates.

Proposition 4. There is a function $\phi: \mathbb{N} \rightarrow \mathbb{N}$ having the following property:

Let $\mathbb{F}_{q}$ be a finite field, $N \geq 2$, and $X \subseteq \mathbb{P}^{N}$ a smooth cubic hypersurface over $\mathbb{F}_{q}$. If $q=\left|\mathbb{F}_{q}\right|>\phi(N)$, then for every $p \in X\left(\mathbb{F}_{q}\right)$, there is a point $x \in X\left(\mathbb{F}_{q}\right)$ such that the line $\ell(x, p)$ joining $x$ and $p$ intersects $X$ at exactly three distinct $\mathbb{F}_{q}$-points.

Proof. Fix $p \in X\left(\mathbb{F}_{q}\right)$. Set

$$
B=\left\{x \in X\left(\mathbb{F}_{q}\right) \mid x \neq p \text { and } \ell(x, p) \nsubseteq T_{p} X\right\},
$$

where $T_{p} X \subseteq \mathbb{P}^{N}$ denotes the tangent hyperplane to $X$ at $p$. For a line $\ell \subseteq \mathbb{P}^{N}$ defined over $\mathbb{F}_{q}$ not contained in $X$ and a point $x \in(\ell \cap X)\left(\mathbb{F}_{q}\right)$, we denote by $(\ell \cdot X)_{x}$ the intersection multiplicity of $\ell$ with $X$ at the point $x$. Then $B=B_{1} \cup B_{2}$, where for $i=1,2$, the subset $B_{i}$ consists of points $x \in B$ such that $(\ell(x, p) \cdot X)_{x}=i$. Let $b=\# B$ and $b_{i}=$ $\# B_{i}, i=1,2$. We have

$$
\begin{aligned}
b= & b_{1}+b_{2}=\#\left(X\left(\mathbb{F}_{q}\right) \backslash\{p\}\right)- \\
& \#\left\{\mathbb{F}_{q} \text {-line } \ell \subseteq T_{p} X \mid p \in \ell \text { and }(\ell \cdot X)_{p}=2\right\}
\end{aligned}
$$

whence

$$
\# X\left(\mathbb{F}_{q}\right)-1-\frac{q^{N-1}-1}{q-1} \leq b=b_{1}+b_{2} \leq \# X\left(\mathbb{F}_{q}\right)-1 .
$$

We want to show that for $q$ large enough,

$$
\# X\left(\mathbb{F}_{q}\right)-1-\frac{q^{N-1}-1}{q-1}>b_{2}
$$


so that $B_{1} \neq \emptyset$. Observe that

$$
B_{2}=\left\{x \in X\left(\mathbb{F}_{q}\right) \mid x \notin T_{p} X, p \in T_{x} X\right\} .
$$

Suppose that $X$ is defined by a cubic form $\varphi \in \mathbb{F}_{q}\left[T_{0}, \cdots, T_{N}\right]$ and put $\varphi_{0}^{\prime}=\frac{\partial \varphi}{\partial T_{0}}$. We may assume that $p=(1: 0: \cdots: 0)$. Letting $Y \subseteq \mathbb{P}^{N}$ denote the subscheme defined by the equations $\varphi=\varphi_{0}^{\prime}=0$, we have clearly $B_{2} \subseteq Y\left(\mathbb{F}_{q}\right)$, whence $b_{2} \leq \# Y\left(\mathbb{F}_{q}\right)$.

By the Lang-Weil theorem,

$$
\# X\left(\mathbb{F}_{q}\right)=q^{N-1}+O\left(q^{N-3 / 2}\right)
$$

where the term $O\left(q^{N-3 / 2}\right)$ depends only on $N$ and $q$. Using [9], Théorème 8.1, we can get an even more explicit estimate:

$$
\left|\# X\left(\mathbb{F}_{q}\right)-\frac{q^{N}-1}{q-1}\right| \leq \beta_{N} \cdot q^{\frac{N-1}{2}}
$$

where $\beta_{N}$ denotes the $(N-1)$-th Betti number of a smooth cubic hypersurface in $\mathbb{P}_{\mathbb{C}}^{N}$. In view of (B) , it is now sufficient to show that

$$
\# Y\left(\mathbb{F}_{q}\right)=O\left(q^{N-2}\right)
$$

with the term $O\left(q^{N-2}\right)$ depending only on $N$ and $q$. An easy verification shows that the form $\varphi_{0}^{\prime}$ cannot be identically zero on $X$ since $X$ is smooth and geometrically irreducible. It follows that $\operatorname{dim} Y<\operatorname{dim} X=$ $N-1$ and thus (4) follows from the classical Lang-Weil bound. In our case, it is also possible to give an explicit bound for $\# Y\left(\mathbb{F}_{q}\right)$. For instance, [30], Chapter 4, Lemma 3.3 gives

$$
\# Y\left(\mathbb{F}_{q}\right) \leq \frac{12 q^{N-1}}{q-1}
$$

The proposition is thus proved.

Remark 3. According to the above proof (cf. (3) and (5)), for a function $\phi: \mathbb{N} \rightarrow \mathbb{N}$ to have the property in Proposition 4 , one sufficient condition is

$$
\frac{12 q^{N-1}}{q-1}<q^{N-1}-1-\beta_{N} q^{\frac{N-1}{2}}, \quad \forall N \geq 2, \forall q>\phi(N) .
$$

So one can compute explicitly at least one possible value of $\phi(N)$ for each $N$. For instance, using $\beta_{3}=7$ one verifies easily that one may take $\phi(3)=20$.

Remark 4 . Letting $\mathscr{L}_{N}$ be the set of $\mathbb{F}_{q}$-lines $\ell \subseteq \mathbb{P}^{N}$ such that

$$
\ell \cap X=\left\{p, x,{ }^{\sigma} x\right\}, \quad \text { with } x \in X\left(\mathbb{F}_{q^{2}}\right) \backslash X\left(\mathbb{F}_{q}\right),
$$

our proof of Proposition 4 can also give a lower bound depending on $N$ (and $q$ ) for the cardinality $\# \mathscr{L}_{N}$. But as a sufficient condition for $\mathscr{L}_{N} \neq \emptyset$, Kollár's lemma (Lemma 4) is obviously better. 
Lemma 5. Let $\widetilde{V}$ be a smooth cubic surface over a finite field $k$ of characteristic not dividing 6 . If $q=|k|>47$, then for any $\widetilde{P} \in \widetilde{V}(k)$, one can find a point $\widetilde{R} \in \widetilde{V}(k)$ satisfying all the conditions (1), (2) and (3) of Theorem 8 .

Proof. Swinnerton-Dyer has noticed this fact as he remarked in [31], p.379, lines 7-10. However, an explicit proof seems not included there. So we give our own proof here.

Let $B_{1}$ be the set of points $\widetilde{R} \in \widetilde{V}(k)$ for which the condition (1) holds. We know from the proof of Proposition 4 (cf. (3) and (5) ) that the cardinality $b_{1}=\# B_{1}$ satisfies

$$
b_{1} \geq \frac{q^{3}-1}{q-1}-\beta_{3} q-\left(1+\frac{13 q^{2}-1}{q-1}\right)=q^{2}-6 q-\frac{13 q^{2}-1}{q-1} .
$$

Since the union of all geometric lines on $\widetilde{V}$ contains at most $27(q+1)$ $k$-points, the subset $B_{1}^{\prime} \subseteq B_{1}$ consisting of points $\widetilde{R} \in \widetilde{V}(k)$ which have both the properties (1) and (2) has cardinality

$$
b_{1}^{\prime}=\# B_{1}^{\prime} \geq b_{1}-27(q+1) \geq q^{2}-46 q-52 .
$$

For $q>47$, we can always find a point $\widetilde{R} \in \widetilde{V}(k)$ having the properties (1) and (2). Then the intersection $\gamma=T_{\widetilde{R}} \widetilde{V} \cap \widetilde{V}$ is a geometrically irreducible plane cubic curve. Such a curve has at least $\underset{\widetilde{V}}{q-2}$ smooth rational points. The points $t \in \widetilde{V}(k)$ such that $\widetilde{P} \in T_{t} \widetilde{V}$ all lie on a quadratic $Y$ and the intersection $\gamma \cap Y$ hat at most 6 points. Thus, when $q>47$, we can always find points $t_{1} \neq t_{2} \in \gamma(k)$ with $t_{i} \neq \widetilde{R}$ such that the line $\widetilde{P} t_{i}$ intersects $\widetilde{V}$ at 3 distinct points for each $i=1,2$. It remains to show that $t_{1}, t_{2}$ may be so chosen that the intersection

$$
T_{t_{1}} \gamma \cap T_{t_{2}} \gamma \cap T_{\widetilde{P}} \widetilde{V}=T_{t_{1}} \widetilde{V} \cap T_{t_{2}} \widetilde{V} \cap\left(T_{\widetilde{R}} \widetilde{V} \cap T_{\widetilde{P}} \widetilde{V}\right)
$$

is empty. This is a consequence of the Lemma 6 below.

Lemma 6. Let $F$ be any field of characteristic not dividing $6, \gamma \subseteq \mathbb{P}_{F}^{2}$ a geometrically irreducible plane cubic curve having a nonsmooth point $R \in \gamma(F)$. Then for any $S \in \mathbb{P}^{2}(F)$, the set

$$
\mathscr{T}:=\{t \in \gamma(F) \mid \text { the line St joining } S \text { and } t \text { is tangent to } \gamma \text { at } t\}
$$

has cardinality $\leq 6$.

Proof. Take coordinates such that $R=(1: 0: 0)$. Since $\gamma$ passes through $R$ and is not smooth there, the equation of $\gamma$ has the following form

$$
\varphi=T_{0} q\left(T_{1}, T_{2}\right)+c\left(T_{1}, T_{2}\right)
$$


where $q$ is a quadratic form and $c$ is a cubic form. We may assume $S \neq R$ and take coordinates such that $S=(0: 1: 0)$. Then $\mathscr{T}$ is the set of rational points of the subscheme $Z \subseteq \mathbb{P}_{F}^{2}$ defined by

$$
\varphi=\frac{\partial \varphi}{\partial T_{1}}=0
$$

Using the hypothesis on the characteristic of $F$, one concludes by an easy computation that $\frac{\partial \varphi}{\partial T_{1}}$ is not identically zero because of the geometrical irreducibility of $\gamma$. It then follows that $\# \mathscr{T}=\# Z(F) \leq 6$.

Proof of Theorem 5. We apply Theorem 8 with $V$ equal to the generic fiber $X$ and $\widetilde{V}$ equal to the special fiber $\mathcal{X}_{p}$. By Theorem 4 , the hypothesis implies that lifting a point in $\widetilde{V}(k)$ to a point in $V(K)$ is always possible. The result then follows easily from Theorem 8 and Lemma 5

Acknowledgements. The author wishes to thank the referee for valuable comments, especially for pointing out the possibility of generalizing the first version of Theorem 4 using the reference [21]. The author also thanks Prof. Jean-Louis Colliot-Thélène for many valuable discussions and comments. Some of the problems solved here arose out of conversations which Jean-Louis Colliot-Thélène and Brendan Hassett had in 2005, and the approach we follow is also inspired by their discussions. Thanks also go to Prof. János Kollár for useful discussions about cubic hypersurfaces over finite fields.

\section{References}

[1] Campana, F.: Connexité rationnelle des variétés de Fano. Ann. Sci. École Norm. Sup. (4) 25 No.5, 539-545 (1992)

[2] Colliot-Thélène, J.-L.: Groupes linéaires sur les corps de fonctions de courbes réelles. J. für die reine und angew. Math. 474, 139-167 (1996)

[3] Colliot-Thélène, J.-L., Gille, P.: Remarques sur l'approximation faible sur un corps de fonctions d'une variable. In: Poonen, B., Tschinkel, Y.(eds.): Arithmetic of higher-dimensional algebraic varieties. Prog. in Math., 226, 121-134, Birkhäuser, Boston (2003)

[4] Colliot-Thélène, J.-L., Sansuc, J.-J.: La $R$-équivalence sur les tores. Ann. Sci. École Norm. Sup. (4) 10 No.2, 175-229 (1977)

[5] Conduché, D.: Courbes rationnelles et hypersurfaces de l'espace projectif, Thèse de doctorat de l'Université Louis Pasteur, Strasbourg (2006) 
[6] de Jong, A.J., Starr, J.: Every rationally connected variety over the function field of a curve has a rational point. Am. J. Math. 125 No.3, 567-580 (2003)

[7] Debarre, O.: Higher-dimensional Algebraic Geometry. SpringerVerlag, New York (2001)

[8] Debarre, O.: Variétés rationnellement connexes. Séminaire Bourbaki, vol. 2001-2002, Astérisque 290 Expo. No.905, ix, 243-266 (2003)

[9] Deligne, P.: La conjecture de Weil I. Publ. Math. I.H.É.S., 43, 273-307 (1974)

[10] Ducros, A.: L'obstruction de réciprocité à l'existence de points rationnels pour certaines variétés sur le corps des fonctions d'une courbe réelle. J. für die reine angew. Math. 504 73-114 (1998)

[11] Fried, M., Jarden, M.: Field Arithmetic, Springer-Verlag, Berlin (1986)

[12] Graber, T., Harris, J., Starr, J.: Families of rationally connected varieties. J. Am. Math. Soc. 16 No.1, 57-67 (2003)

[13] Grothendieck, A., Dieudonné, J.: Éléments de géométrie algébrique, IV, Étude locale des schémas et des morphismes de schémas, seconde partie. Publ. Math. I.H.É.S. 24, 5-231 (1965).

[14] Hartshorne, R.: Algebraic Geometry. GTM 52, Springer-Verlag, New York (1977)

[15] Hassett, B., Tschinkel, Y.: Weak approximation over function fields. Invent. Math. 163 No.1, 171-190 (2006)

[16] Hassett, B., Tschinkel, Y.: Approximation at places of bad reduction for rationally connected varieties. Pure and Applied Mathematics Quarterly 4 No. 3, 743-766 (2008)

[17] Knecht, A.: Weak approximation for general degree two del Pezzo surfaces. arXiv: 0809.1251

[18] Kollár, J.: Rational Curves on Algebraic Varieties. SpringerVerlag, Berlin (1996)

[19] Kollár, J.: Rationally connected varieties over local fields. Ann. of Math. 150, 357-367 (1999)

[20] Kollár, J.: Specialization of zero cycles. Publ. RIMS, Kyoto Univ. 40, 689-708 (2004) 
[21] Kollár, J.: Looking for rational curves on cubic hypersurfaces. arxiv: $0710.5516 \mathrm{v} 1$

[22] Kollár, J., Miyaoka, Y., Mori, S.: Rationally connected varieties. J. Alg. Geo. 1 No. 3, 429-448 (1992)

[23] Kollár, J., Szabó, E.: Rationally connected varieties over finite fields. Duke Math. J. 120 No.2, 251-267 (2003)

[24] Lang, S., Weil, A.: Number of points of varieties in finite fields. Am. J. Math. 76, 819-827 (1954)

[25] Madore, D.: Approximation faible aux places de bonne réduction sur les surfaces cubiques sur les corps de fonctions. Bull. Soc. Math. France, 134 No. 4, 475-485 (2006)

[26] Nagata, M.: A generalization of the imbedding problem of an abstract variety in a complete variety. J. Math. Kyoto Univ. 3, 89-102 (1963)

[27] Pop, F.: Embedding problems over large fields. Ann. of Math. 144, $1-34(1996)$

[28] Prestel, A., Roquette, P.: Formally p-adic fields. Lecture Notes in Math. vol. 1050, Springer-Verlag, Berlin (1984)

[29] Scheiderer, C.: Hasse principles and approximation theorems for homogeneous spaces over fields of virtual cohomological dimension one. Invent. Math. 125 No. 2, 307-365 (1996)

[30] Schmidt, W.: Equations over finite fields: an elementary approach. Kendrick Press, Heber City (Utah) (2004)

[31] Sir Peter Swinnerton-Dyer: Weak approximation and $R$ equivalence on cubic surfaces. In Peyre, E., Tschinkel, Y. (eds.): Rational points on algebraic varieties. Progress in Mathematics, 199, 357-404, Birkäuser, Boston (2001)

[32] Wittenberg, O.: La connexité rationnelle en arithmétique. http://www.dma.ens.fr/ ${ }^{\sim}$ wittenberg (2008)

[33] Xu, C.: Weak approximation for low degree Del Pezzo surfaces. arXiv: 0902.1765 